\section{Associaccão entre prática religiosa e comportamentos de risco à saúde em adolescentes de Pernambuco, Brasil}

\section{Association between religious practice and health risk behaviors in adolescents of northeast, Brazil}

Ana Raquel Mendes dos Santos ${ }^{1}$

Luciano Machado Ferreira T. Oliveira ${ }^{2}$

José Cazuza de Farias Júnior ${ }^{3}$

Priscilla Pinto Costa da Silva

Emília Amélia Pinto Costa da Silva ${ }^{4}$

Clara Maria Silvestre Monteiro de Freitas ${ }^{5}$

\section{RESUMO}

Objetivou-se analisar a associação entre a religiosidade e os comportamentos de risco à saúde (baixo nível de atividade física, consumo de álcool, drogas ilícitas, tabaco e comportamento sexual), de forma isolada e simultânea, em 6.264 adolescentes do Ensino Médio da Rede Pública no Estado de Pernambuco. Trata-se de estudo epidemiológico transversal que coletou informações por meio do questionário Global School-Based Student Health Survey. Na análise dos dados recorreu-se ao teste de qui-quadrado $\left(\chi^{2}\right)$ e à regressão logística binária. Os resultados revelaram que, entre os rapazes e as moças que estavam expostos aos 5 comportamentos de risco de forma simultânea, 61 e $90 \%(\mathrm{P}<0,001)$, respectivamente, não possuíam ou não praticavam uma religião. Os adolescentes que não têm ou não praticam uma religião possuem mais chances de consumir álcool, cigarros, drogas ilícitas e ter um número elevado de parceiros sexuais, quando comparados aqueles que possuem e praticam uma determinada religião $(\mathrm{P}<0,05)$. Foi observado na análise ajustada que a prática de uma religião, independentemente da religião, foi considerada como fator de proteção para o consumo de álcool, cigarros, drogas ilícitas e número elevado de parceiros sexuais, quando comparados aqueles que tinham, mas não praticavam sua religião, sendo esta associação maior entre as moças que entre os rapazes. Não foi encontrada associação entre a prática religiosa e o nível de atividade física. Conclui-se que a religiosidade, independentemente da religião, mostrou-se associada à proteção aos comportamentos de risco à saúde neste grupo de adolescentes.

\section{PALAVRAS-CHAVE}

Religião; Adolescente; Comportamentos de risco.

\begin{abstract}
This study aimed to analyze the association between religiosity and health risk behaviors (low physical activity, alcobol consumption, illicit drugs, tobacco and sexual behavior), isolated and simultaneously in 6,264 adolescents Middle School Network public in the State of Pernambuco. This cross-sectional epidemiological study collected information through the questionnaire Global School-based Student Health Survey. In the data analysis was done using the chi-square test $(\chi 2)$ and binary logistic regression. The results revealed that among the young men and women who were exposed to 5 risk behaviors simultaneously, 61 and 90\% (P<0.001), respectively, did not bave or did not practice a religion. Adolescents who do not have or do not practice a religion have more chances to consume alcobol, cigarettes, illegal drugs and have a high number of sexual partners, compared those with and practice a particular religion $(P<0.05)$. Was observed in the adjusted analysis that the practice of a religion, then regardless of religion, was considered as a protective factor for the consumption of alcohol, cigarettes, illegal drugs and high number of sexual partners, compared those who had, but did not practice their religion, and this association was greater among girls than among boys. No association was found between religious practice and the level of physical activity. It follows that religion, regardless of which religion was associated with the protection of health risk behaviors in this group of adolescents.
\end{abstract}

Rev Bras Ativ Fís Saúde p. 284-296 DOI

http://dx.doi.org/10.12820/rbafs.v.20n3p284

1 Universidade de Pernambuco. Doutoranda do Programa Associado de Pós-Graduação em Educação Física UPE/UFPB. Recife-PE, Brasil.

2 Universidade Federal de Pernambuco.

Doutorando do Programa de Pós-Graduação em Neuropsiquiatria e Ciências do Comportamento. Recife-PE, Brasil.

3 Universidade Federal da Paraíba. Prof. Dr. do Programa Associado de Pós-Graduação em Educação Física UPE/UFPB. João Pessoa-PB, Brasil.

4 Universidade Federal do Paraná. Doutoranda do Programa de Pós-Graduacão de Pós-Graduacãa em Educação Física UFPR. Curitiba-PR, Brasil.

5 Universidade de Pernambuco. Profa. Dra do Programa Associado de Pós-Graduação em Educação Física UPE/UFPB. Recife, PE, Brasil.

\title{
KEYWORDS
}


A religião pode exercer um papel estabilizador, construtivo e humanizador para a existência humana ${ }^{1}$. Sua definição está pautada em um sistema de orientação e admiração, trazendo consigo sentimentos, atos e experiências ligadas ao que é sagrado. Nessa direção, encontram-se também os mitos, ritos, símbolos e a congregação de pessoas que buscam o mundo espiritual. Assim, possuir afiliação religiosa e praticá-la representa respeitar normas morais sobre como lidar com a vida, com o mundo e com pessoas, oferecendo subsídios que auxiliam de forma positiva na reflexão, compreensão e adoção de atitudes e comportamentos favoráveis à vida humana ${ }^{2}$.

A dimensão religiosa quando presente no cotidiano dos indivíduos, sobretudo entre os jovens, pode representar um fator influenciador para as relações sociais e familiares, apoiados por pressupostos éticos, políticos e culturais. Além disso, algumas práticas religiosas são capazes de proporcionar aspectos salutares que implicam positivamente na saúde física e mental dos adolescentes, na medida em que aconselham a adoção de hábitos e condutas saudáveis. A este respeito, estudos ${ }^{3,4}$ sugerem uma estreita relação entre religiosidade e comportamentos no estilo de vida, sugerindo que tanto a afiliação quanto a prática religiosa poderiam ser fatores que influenciariam na não adoção de comportamentos de risco à saúde dos adolescentes.

Sabendo que a adolescência é um período no qual ocorrem intensas mudanças na vida dos jovens, é necessário considerar que nesta fase há diversidades de conflitos decorridos do processo de enfrentamento das modificações biológicas, cognitivas, emocionais e sociais. Essa fase também se caracteriza pelo crescimento da autonomia e independência em relação à família, resultando na exploração e experimentação de novos comportamentos e vivências que podem apresentar consequências negativas, tais como níveis insuficientes de atividade física, consumo de álcool, drogas ilícitas, tabagismo e elevado números de parceiros sexuais ${ }^{5}$.

Estes comportamentos de risco estão, direta ou indiretamente, associados à maior taxa de morbimortalidade, doenças sexualmente transmissíveis e gravidez indesejada, além das doenças crônico-degenerativas não transmissíveis ${ }^{6}$. Em função disso, estudos sobre comportamentos de risco têm sido desenvolvidos com adolescentes e estima-se que $6 \%$ a $65,1 \%$ deles estejam expostos a um ou mais comportamentos de risco à saúde ${ }^{3,7-9}$. Nesse sentido, identificar os fatores que podem influenciar ou que estão associados à adoção desses comportamentos representa uma informação importante para o desenvolvimento de ações de intervenção. Dentre esses fatores, acredita-se que a dimensão religiosa pode estar associada a não adoção dos comportamentos de risco em adolescentes, tendo em vista que a afiliação a uma religião traz consigo valores e normas sociais que influenciam positivamente no estilo de vida ${ }^{4}$.

Vale salientar que há carência de estudos abordando essa relação em adolescentes. Portanto, este estudo objetivou analisar a associação entre a religiosidade e os comportamentos de risco à saúde (consumo de álcool, drogas ilícitas, tabaco, envolvimento com dois ou mais parceiros sexuais e nível insuficiente de atividade física), de forma isolada e simultânea, em adolescentes.

\section{MÉTODOS}

Trata-se de um estudo descritivo que integra o levantamento epidemiológico 
transversal de base escolar e abrangência estadual intitulado "Prática de atividades físicas e comportamentos de risco à saúde em estudantes do Ensino Médio no Estado de Pernambuco: Estudo de tendência temporal (2006-2011)".

A população-alvo foi estudantes da rede pública de ensino médio de Pernambuco, com 14 a 19 anos de idade. Para cálculo do tamanho amostral foram adotados os parâmetros: população estimada em 373.386 estudantes; intervalo de confiança de $95 \%$; erro máximo tolerável de 2 pontos percentuais; prevalência estimada em 50\%; efeito de delineamento amostral estabelecido em 2 vezes o tamanho mínimo da amostra. Houve acréscimo de $20 \%$ no tamanho da amostra em cada região para eventuais perdas na aplicação/preenchimento inadequado do questionário, resultando em 5.683 escolares.

Objetivando estimar o poder estatístico das análises empregadas, realizouse cálculos a posteriori utilizando o Epi Info 7. Verificou-se que o quantitativo de indivíduos do sexo masculino permitiu detectar como significativos valores de OR entre 0,57 e 0,82 , com a prevalência dos desfechos variando de 3,8\% a $70,8 \%$ nos expostos; para o sexo feminino, o número de participantes detectou como estatisticamente significativos valores de OR entre 0,57 e 0,80 , com prevalência do desfecho variando de $5,9 \%$ a $56,1 \%$ nos expostos; com poder estatístico de $80 \%$ e nível de confiança de $95 \%$.

Para garantir que a amostra selecionada representasse a população alvo, considerou-se: tamanho das escolas de pequeno (menos de 200 alunos), médio (200-499 alunos) e grande porte (500 alunos ou mais) e distribuição dos estudantes quanto ao turno de estudo (diurno e noturno). Os alunos matriculados no período da manhã e da tarde foram agrupados em uma única categoria (período diurno). A distribuição regional considerou o número de escolas existentes em cada uma das 17 Gerências Regionais de Educação (GREs).

$\mathrm{Na}$ seleção dos sujeitos recorreu-se a amostragem aleatória estratificada por conglomerados em dois estágios, sendo a "escola" e a "turma" as unidades amostrais no primeiro e no segundo estágio, respectivamente. No primeiro estágio, adotou-se como critério de estratificação a densidade das escolas em cada GRE do Estado e seu porte. No segundo estágio, considerou-se a densidade de turmas nas escolas sorteadas por período de estudo (diurno e noturno) e séries de ensino ( $1^{\mathrm{a}}, 2^{\mathrm{a}}$ e $3^{\mathrm{a}}$ do ensino médio).

A coleta dos dados foi realizada de maio a novembro de 2011. Utilizouse uma versão traduzida e adaptada do Global School-Based Student Health Survey (GSHS), construído e validado para a população de adolescentes. Os estudantes das turmas sorteadas foram convidados a participar do estudo, independentemente da idade. A aplicação dos questionários foi efetuada em sala de aula, sem a presença dos professores e os estudantes foram assistidos por dois aplicadores para que pudessem esclarecer dúvidas. O tempo para preenchimento do instrumento durou aproximadamente 40-50 minutos.

Antes do início da coleta de dados, um estudo piloto foi conduzido a fim de testar a aplicabilidade do instrumento. Os dados foram coletados em uma escola de referência da rede pública estadual de ensino de Recife, com 86 adolescentes apresentando idade de 14 a 19 anos. Os indicadores de reprodutibilidade apresentaram coeficiente de correlação intraclasse de moderados a altos na maioria dos itens do questionário, tendo os coeficientes de concordância (índice kappa) variado de 0,52 a 1,00.

As variáveis sociodemográficas mensuradas neste estudo foram: "idade" (14-16 anos; 17-19 anos), "local de residência" (urbano e rural), "afiliação re- 
ligiosa" (não tem religião, católica, evangélica, espírita e outra) e "nível socioeconômico" (elevado ou baixo). Em relação ao nível socioeconômico, foi utilizado a escolaridade materna, sendo estratificada em menor ou igual a 8 anos de estudo e maior a 8 anos de estudo.

Os comportamentos de risco à saúde (variáveis dependentes) analisados foram: níveis insuficientes de atividade física, consumo de álcool, drogas ilícitas, tabaco e número elevado de parceiros sexuais. Em relação a variável "nível de atividade física", foram consideradas duas questões do GSHS: 1) "Durante os últimos 7 dias, quantos dias você foi fisicamente ativo por um total de pelo menos 60 minutos por dia?" e 2) "Durante uma semana típica ou normal, em quantos dias você é fisicamente ativo por um total de pelo menos 60 minutos ao dia?”. Para verificar o nível de atividade física, adotou-se o procedimento sugerido por Prochaska, Sallis e Long ${ }^{10}$ referente às duas questões anteriores, utilizando a fórmula: (questão $1+$ questão 2$) \div 2$. Resultados inferiores a cinco dias foi utilizado para classificar os adolescentes como insuficientemente ativos ${ }^{11}$.

A variável "consumo de álcool" foi determinada mediante análise das respostas à questão: "Nos últimos 30 dias, em quantos dias você consumiu pelo menos uma dose de bebida contendo álcool?". A variável "consumo de tabaco" foi identificada mediante as respostas da pergunta: "Durante os últimos 30 dias, em quantos dias você fumou cigarros?”. Foram considerados expostos aqueles que relataram consumir bebida alcoólica e fumo, em pelo menos uma vez nos últimos trinta dias, independentemente da quantidade 3 . A variável "consumo de drogas" foi verificada diante da questão: "Durante a sua vida, quantas vezes você utilizou drogas, tais como: loló, cola de sapateiro, lança perfume, maconha, crack, cocaína ou outras (não considerar cigarro ou bebida alcoólica)?”. Considerou-se exposto o relato de uso durante a vida de alguma dessas drogas ${ }^{15}$. Relacionado ao número de parceiros sexuais, os adolescentes que já iniciaram sua vida sexualmente ativa, responderam a pergunta: "Durante a sua vida, com quantas pessoas você já teve relação sexual?”. Esta variável foi dicotomizada em "até uma pessoa" e "duas pessoas ou mais"12.

A variável independente do estudo foi "afiliação/prática religiosa". Assim, os adolescentes responderam as questões: "Qual a sua religião?" e "Você se considera praticante da sua religião?". A variável "afiliação religiosa" tinha cinco opções de respostas: não tenho religião, católica, evangélica, espírita e outra. Esta variável foi dicotomizada em "possui afiliação" e "não possui filiação religiosa". A variável "prática religiosa" tinha duas opções de respostas: "sim" e "não". Para fins de análise, as variáveis "afiliação" e "prática religiosa" foram agrupadas, recebendo a denominação: "afiliação/prática religiosa", com as categorias: "não tem ou não pratica a religião" e "tem e pratica a religião".

Além disso, foram analisados os comportamentos de risco de forma simultânea considerando os adolescentes que tinham dados completos para os cinco comportamentos de risco analisados, que somados, obtiveram valor variando de "zero" (para aqueles que não apresentaram nenhum comportamento) a cinco (para aqueles expostos a todos os comportamentos). Foram avaliados os adolescentes com "nenhum ou 1", “ 2 ou 3" e "4 ou 5" comportamentos de risco à saúde (baixo nível de atividade física, consumo de álcool, drogas ilícitas, tabaco e comportamento sexual de risco).

A tabulação dos dados foi efetuada no EpiData (versão 3.1). Recorreu-se à dupla digitação a fim de conferir erros na entrada dos dados que, quando 
identificados, foram corrigidos com base nos valores originais das variáveis. A análise dos dados realizou-se através do SPSS 10.0. Na análise descritiva foi observada a distribuição de frequências das variáveis dependentes, independente e das variáveis do perfil demográfico, socioeconômico e religioso. $\mathrm{Na}$ análise inferencial, utilizou-se o teste de qui-quadrado $\left(\chi^{2}\right)$ para comparar a prevalência de adolescentes expostos a diferentes quantidades de comportamentos de risco à saúde, entre os que possuíam e praticavam a religião e aqueles que não possuíam ou não praticavam a religião. A regressão logística binária bruta e ajustada foi utilizada para avaliar a associação entre os comportamentos de risco à saúde e a "afiliação/prática religiosa". Desse modo, elaborou-se cinco modelos de análise, em que cada modelo a variável dependente foi o comportamento de risco à saúde (baixo nível de atividade física, consumo de álcool, drogas ilícitas, tabaco e comportamento sexual de risco) e a variável independente foi afiliação/prática religiosa. As variáveis "idade", "local de residência", "nível socioeconômico" e "afiliação religiosa", foram consideradas como possíveis fatores de confusão. Entraram no modelo apenas àquelas que obtiveram nível de significância estatística menor que $0,20(\mathrm{p}<0,20)$. Decidiu-se estratificar a análise por sexo, uma vez que identificou-se a presença de interação com a afiliação/prática religiosa. Para todos os testes o nível de significância adotado foi $\mathrm{p}<0,05$.

O estudo foi aprovado pelo Comitê de Ética em Pesquisa com Seres Humanos da Universidade de Pernambuco (CAAE-0158.0.097.000-10/CEP-UPE: 159/10), respeitando a normatização da resolução 196/96.

\section{RESULTADOS}

Foram entrevistados 7.195 estudantes de 85 escolas em 48 municípios pernambucanos. As recusas totalizaram 333 (4,6\%), sendo 317 por parte dos adolescentes e 16 dos pais. Após a exclusão dos questionários dos estudantes com idade inferior a 14 e superior a 19 anos, a amostra final foi 6.264 , os quais $59,7 \%$ eram do sexo feminino com média de idade de 16,6 anos ( $\mathrm{DP}= \pm 1,3$ anos).

Na tabela 1 são apresentadas as características socioeconômicas, sociodemográficas, afiliação e prática religiosa e a prevalência de comportamentos de risco, estratificado por sexo.

A maioria dos adolescentes tinha idade entre 17 a 19 anos (51,6\%), era solteiro $(91,4 \%)$, residia na região urbana $(74,5 \%)$ e tinha o nível socioeconômico mais baixo (86,1\%). Foi observado que $86,5 \%$ dos jovens possuíam uma afiliação religiosa $(86,5 \%)$, sendo católica $(56 \%)$ e evangélica $(26,9 \%)$ as mais indicadas. Os que possuíam afiliação, $74,4 \%$ praticavam sua religião. Verificou-se que 28,6\% consumiam bebidas alcoólicas, 6,6\% usavam drogas ilícitas, 4,6\% fumavam; $27,9 \%$ estavam envolvidos com dois ou mais parceiros sexuais e $64,9 \%$ eram insuficientemente ativos.

As figuras 1 e 2 apresentam a comparação da proporção de adolescentes expostos a "nenhum ou um", "dois ou três" e "quatro ou cinco" comportamentos de risco entre adolescentes que possuíam e praticavam a religião e aqueles que não possuíam ou não praticavam a religião.

A análise das figuras 1 e 2 revelou que a exposição simultânea aos comportamentos de risco foi mais elevada nos adolescentes que não possuíam ou não praticavam a religião, em ambos os sexos. Foi observada, entre os rapazes e as moças que estavam expostos a 4 ou 5 comportamentos de risco, uma maior 
TABELA 1 - Características dos adolescentes, conforme o sexo. Pernambuco, Brasil, 2011/ Characteristics of adolescents, according to sex. Pernambuco, Brazil, 2011

\begin{tabular}{|c|c|c|c|c|c|c|}
\hline \multirow{3}{*}{ Variáveis } & \multicolumn{2}{|c|}{ Masculino } & \multicolumn{2}{|c|}{ Feminino } & \multicolumn{2}{|c|}{ Total } \\
\hline & \multicolumn{2}{|c|}{$2524(40,3 \%)$} & \multicolumn{2}{|c|}{$3737(59,7 \%)$} & \multicolumn{2}{|c|}{6261} \\
\hline & $\mathrm{n}$ & $\%$ & $n$ & $\%$ & $\mathrm{n}$ & $\%$ \\
\hline \multicolumn{7}{|l|}{ Idade (anos) } \\
\hline $14-16$ & 1109 & 43,9 & 1919 & 51,4 & 3028 & 48,4 \\
\hline $17-19$ & 1415 & 56,1 & 1818 & 48,6 & 3233 & 51,6 \\
\hline \multicolumn{7}{|l|}{ Estado civil } \\
\hline Solteiro (a) & 2325 & 93,4 & 3335 & 89,9 & 5660 & 91,3 \\
\hline Casado (a)/vivendo com parceiro (a) & 63 & 2,5 & 226 & 6,1 & 289 & 4,7 \\
\hline Outro & 104 & 4,1 & 149 & 4,0 & 253 & 4,1 \\
\hline \multicolumn{7}{|l|}{ Local de residência } \\
\hline Urbano & 1878 & 74,6 & 2766 & 74,5 & 4644 & 74,5 \\
\hline Rural & 640 & 25,4 & 947 & 25,5 & 1587 & 25,5 \\
\hline \multicolumn{7}{|l|}{ Nível socioeconômico } \\
\hline Elevado & 266 & 12,3 & 479 & 15,1 & 745 & 13,9 \\
\hline Baixo & 1903 & 87,7 & 2694 & 84,9 & 4597 & 86,1 \\
\hline \multicolumn{7}{|l|}{ Afiliação religiosa } \\
\hline Não tem religião & 436 & 17,3 & 406 & 10,9 & 842 & 13,5 \\
\hline Católica & 1262 & 50,2 & 2239 & 60,0 & 3501 & 56,0 \\
\hline Evangélica & 701 & 27,9 & 983 & 26,3 & 1684 & 26,9 \\
\hline Espírita & 43 & 1,7 & 39 & 1,0 & 82 & 1,3 \\
\hline Outra & 74 & 2,9 & 67 & 1,8 & 141 & 2,3 \\
\hline \multicolumn{7}{|l|}{ Prática religiosa* } \\
\hline Sim & 1431 & 56,9 & 2591 & 69,4 & 4022 & 74,4 \\
\hline Não & 646 & 25,7 & 735 & 19,7 & 1381 & 25,6 \\
\hline \multicolumn{7}{|l|}{ Consumo de álcool } \\
\hline Consumiu pelo menos 1 vez nos últimos 30 dias** & 794 & 31,5 & 994 & 26,6 & 1788 & 28,6 \\
\hline Nunca consumiu & 1725 & 68,5 & 2741 & 73,4 & 4466 & 71,4 \\
\hline \multicolumn{7}{|l|}{ Consumo de drogas ilícitas } \\
\hline Consumiu pelo menos 1 vez na vida** & 233 & 9,2 & 182 & 4,9 & 415 & 6,6 \\
\hline Nunca consumiu & 2286 & 90,8 & 3554 & 95,1 & 5840 & 93,4 \\
\hline \multicolumn{7}{|l|}{ Consumo de tabaco } \\
\hline Consumiu pelo menos 1 vez nos últimos 30 dias** & 147 & 5,9 & 142 & 3,8 & 289 & 4,6 \\
\hline Nunca consumiu & 2353 & 94,1 & 3582 & 96,2 & 5935 & 95,4 \\
\hline \multicolumn{7}{|l|}{ Número de parceiros sexuais } \\
\hline Duas pessoas ou mais** & 1267 & 50,5 & 474 & 12,7 & 1741 & 27,9 \\
\hline Até uma pessoa & 1242 & 49,5 & 3259 & 87,3 & 4501 & 72,1 \\
\hline \multicolumn{7}{|l|}{ Nível de atividade física } \\
\hline$<5$ dias** & 1411 & 56,1 & 2634 & 70,8 & 4045 & 64,9 \\
\hline$>=5$ dias & 1103 & 43,9 & 1088 & 29,2 & 2191 & 35,1 \\
\hline
\end{tabular}

*Entre os que possuíam afiliação religiosa. **Exposto ao comportamento de risco. a Entre os que já iniciaram a vida sexual. 
prevalência para aqueles que não possuíam ou não praticavam a religião, $(7,3$ Vs 2,9$)$ e $(5,3$ Vs $0,9 \%)$ respectivamente $(\mathrm{P}<0,001)$.

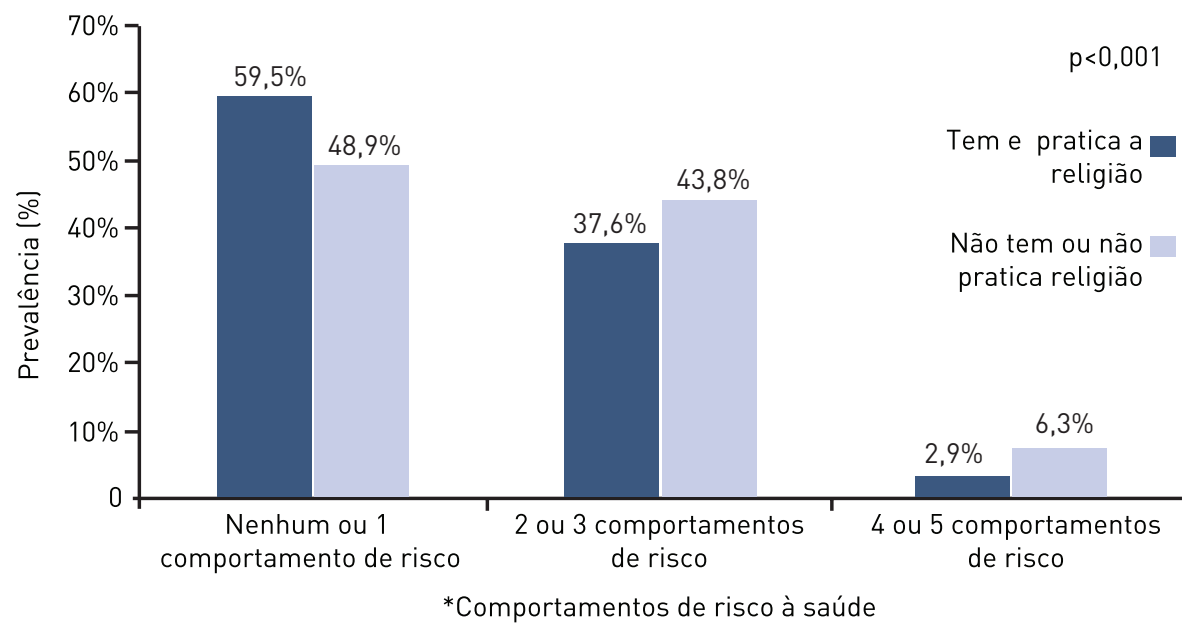

FIGURA 1 - Prevalência de comportamentos de risco à saúde de forma simultânea entre adolescentes do sexo masculino que têm e pratica uma religião e que não têm ou não pratica a religião. Pernambuco, Brasil, 2011.

Comportamentos de risco à saúde: baixo nível de atividade física, consumo de álcool, drogas ilícitas, tabaco e comportamento sexual de risco

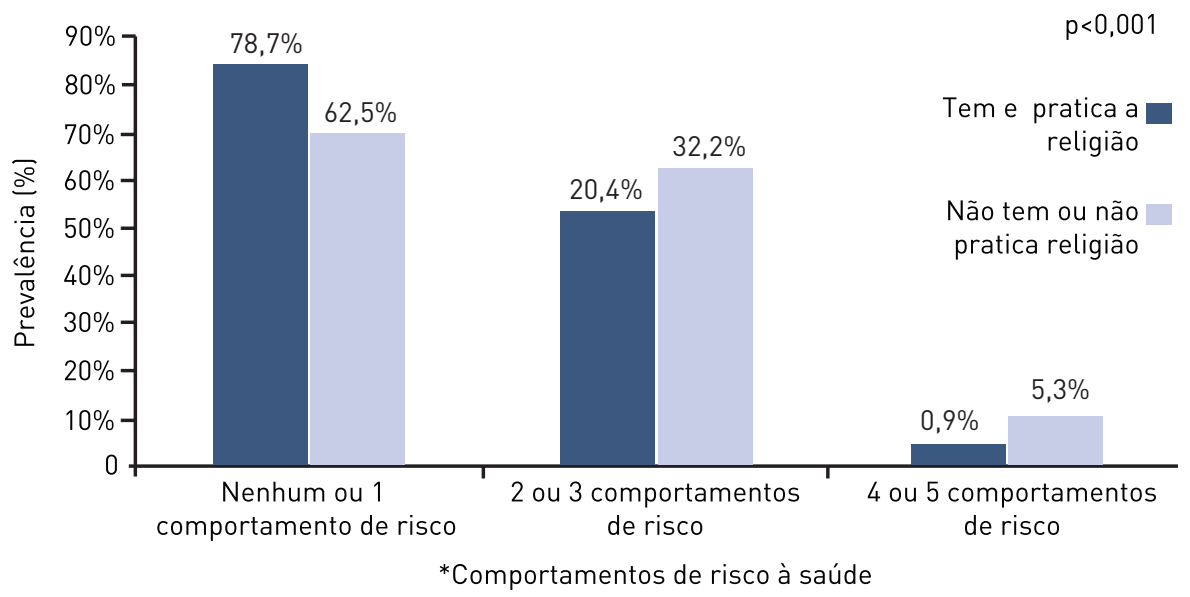

FIGURA 2 - Prevalência de comportamentos de risco à saúde de forma simultânea entre adolescentes do sexo feminino que têm e pratica uma religião e que não têm ou não pratica a religião. Pernambuco, Brasil, 2011/ Prevalence of health risk behaviors simultaneously among female adolescents who have and practice a religion and who do not have or do not practice the religion. Pernambuco, Brazil, 2011

*Comportamentos de risco à saúde: baixo nível de atividade física, consumo de álcool, drogas ilícitas, tabaco e comportamento sexual de risco

Na tabela 2, são apresentados os resultados da análise de regressão logística para avaliar a associação entre afiliação/prática religiosa e os comportamentos de risco em adolescentes do sexo masculino e feminino, respectivamente.

Os resultados da análise ajustada demonstraram que os adolescentes que não têm uma religião ou têm e não praticam uma religião possuem mais chances de consumir álcool, cigarros, drogas ilícitas e ter um número elevado de parceiros sexuais, quando comparados aqueles que possuem e praticam uma determinada religião, isso para os adolescentes de ambos os sexos, independentemente da idade, local de residência e escolaridade da mãe. Não foi encontrada associação entre o nível de atividade física e a afiliação e prática religiosa. 
Na tabela 3, são apresentados os resultados da análise de regressão logística para avaliar a associação entre a prática religiosa e os comportamentos de risco em adolescentes do sexo masculino e feminino, após o ajustamento pela afiliação religiosa.

Os resultados da análise ajustada demonstraram que a prática de uma religião, independentemente da religião seguida, foi considerada como fator de proteção para o consumo de álcool, cigarros, drogas ilícitas e número elevado de parceiros sexuais, quando comparados aqueles que tinham, mas não praticavam sua religião, isso para os adolescentes de ambos os sexos, no entanto, foi obser-

TABELA 2 - Regressão logística ajustada para associação entre afiliação/prática religiosa e os cinco desfechos de comportamentos de risco em adolescentes do sexo masculino e feminino. Pernambuco, Brasil, 2011/ Crude and adjusted logistic regression for the association between affiliation / religious practice and the five outcomes of risk behavior in adolescent males and females. Pernambuco, Brazil, 2011

\begin{tabular}{|c|c|c|c|c|c|}
\hline \multirow[b]{2}{*}{ Indicadores de religiosidade } & \multicolumn{5}{|c|}{ Comportamentos de risco } \\
\hline & $\begin{array}{c}\text { Exposto ao consumo } \\
\text { de álcool }\end{array}$ & $\begin{array}{c}\text { Exposto ao } \\
\text { consumo de tabaco }\end{array}$ & $\begin{array}{l}\text { Exposto ao consumo } \\
\text { de drogas ilícitas }\end{array}$ & $\begin{array}{c}\text { Dois ou mais } \\
\text { parceiros sexuais }\end{array}$ & $\begin{array}{c}\text { Insuficientemente } \\
\text { ativo }\end{array}$ \\
\hline \multicolumn{6}{|l|}{ Masculino } \\
\hline \multirow{2}{*}{ Afiliação e prática religiosa } & OR (IC95\%) & OR (IC95\%) & OR (IC95\%) & OR (IC95\%) & OR (IC95\%) \\
\hline & ajustada $^{a}$ & ajustada $^{a}$ & ajustada $^{a}$ & ajustada $^{a}$ & ajustada $^{a}$ \\
\hline Tem e Pratica a religião & 1 & 1 & 1 & 1 & 1 \\
\hline Tem religião, mas não a pratica & $1,70(1,37-2,10)^{*}$ & $2,08(1,37-3,16)^{*}$ & $1,67(1,19-2,35)^{*}$ & $1,55(1,26-1,90)^{*}$ & $0,97(0,70-1,18)$ \\
\hline Não tem religião & $1,40(1,09-1,80)^{*}$ & $2,03(1,25-3,28)^{*}$ & $2,30(1,59-3,38)^{*}$ & $1,73(1,36-2,21) *$ & $0,94(0,75-1,20)$ \\
\hline \multicolumn{6}{|l|}{ Feminino } \\
\hline \multirow{2}{*}{ Afiliação e prática religiosa } & OR (IC95\%) & OR (IC95\%) & OR (IC95\%) & OR (IC95\%) & OR (IC95\%) \\
\hline & ajustada $^{a}$ & ajustada $^{a}$ & ajustada $^{a}$ & ajustada $^{a}$ & ajustada $^{a}$ \\
\hline Tem e Pratica a religião & 1 & 1 & 1 & 1 & 1 \\
\hline Tem religião, mas não a pratica & $1,96(1,63-2,37)^{*}$ & $2,71(1,78-4,17)^{*}$ & $3,25(2,23-4,74)^{*}$ & $2,80(2,20-3,55)^{*}$ & $1,11(0,92-1,36)$ \\
\hline Não tem religião & $1,90(1,48-2,41)^{*}$ & $5,06(3,24-7,90)^{*}$ & $5,71(3,84-8,50)^{*}$ & $2,74(2,03-3,71)^{*}$ & $1,27(0,98-1,66)$ \\
\hline
\end{tabular}

${ }^{*} p<0,05$

${ }^{a}$ Análise ajustada por idade, local de residência e escolaridade materna.

vada uma maior associação, em todos os comportamentos, entre as moças. Não foi encontrada associação entre a prática religiosa e o nível de atividade física.

TABELA 3 - Regressão logística ajustada pela prática religiosa para associação entre a prática religiosa e os cinco desfechos de comportamentos de risco em adolescentes do sexo masculino e feminino. Pernambuco, Brasil, 2011/ Crude and adjusted logistic regression for the association between affiliation / religious practice and the five outcomes of risk behavior in adolescent males and females. Pernambuco, Brazil, 2011

\begin{tabular}{|c|c|c|c|c|c|}
\hline \multirow[b]{2}{*}{$\begin{array}{l}\text { Indicadores de } \\
\text { religiosidade }\end{array}$} & \multicolumn{5}{|c|}{ Comportamentos de risco } \\
\hline & $\begin{array}{c}\text { Exposto ao consumo } \\
\text { de álcool }\end{array}$ & $\begin{array}{c}\text { Exposto ao consumo } \\
\text { de tabaco }\end{array}$ & $\begin{array}{c}\text { Exposto ao consumo } \\
\text { de drogas ilícitas }\end{array}$ & $\begin{array}{c}\text { Dois ou mais } \\
\text { parceiros sexuais }\end{array}$ & $\begin{array}{c}\text { Insuficientemente } \\
\text { ativo }\end{array}$ \\
\hline \multicolumn{6}{|l|}{ Masculino } \\
\hline \multirow{2}{*}{ Prática religiosa } & OR (IC95\%) & OR (IC95\%) & OR (IC95\%) & OR (IC95\%) & OR (IC95\%) \\
\hline & ajustada $^{a}$ & ajustada $^{a}$ & ajustada $^{a}$ & ajustada $^{a}$ & ajustada $^{a}$ \\
\hline Não & 1 & 1 & 1 & 1 & 1 \\
\hline Sim & $0,64(0,51-0,80)^{*}$ & $0,50(0,33-0,77)^{*}$ & $0,61(0,43-0,86)^{*}$ & $0,67(0,55-0,83)^{*}$ & $1,03(0,84-1,26)$ \\
\hline \multicolumn{6}{|l|}{ Feminino } \\
\hline \multirow{2}{*}{ Prática religiosa } & OR (IC95\%) & OR (IC95\%) & OR (IC95\%) & OR (IC95\%) & OR (IC95\%) \\
\hline & ajustada $^{a}$ & ajustada $^{a}$ & ajustada $^{a}$ & ajustada $^{a}$ & ajustada $^{a}$ \\
\hline Não & 1 & 1 & 1 & 1 & 1 \\
\hline Sim & $0,55(0,46-0,67)^{*}$ & $0,39(0,25-0,60)^{*}$ & $0,31(0,21-0,46)^{*}$ & $0,36(0,28-0,46)^{*}$ & $0,90(0,74-1,10)$ \\
\hline
\end{tabular}

a Análise ajustada pela afiliação religiosa, idade, local de residência e escolaridade materna. 


\section{DISCUSSÃO}

Os principais achados deste estudo estão expostos a seguir: a) foi observada uma alta prevalência referente aos comportamentos de risco à saúde entre os jovens; b) identificou-se também que os jovens que tinham e praticavam uma religião estavam menos expostos aos comportamentos de risco isolados e simultâneos; c) os adolescentes que não tinham ou tinham e não praticavam uma religião consumiam mais álcool, cigarros, drogas e já tiveram um número elevado de parceiros sexuais; d) os adolescentes que praticavam os hábitos fomentados pela religião seguida, independentemente da religião, consumiam menos álcool, cigarros, drogas e tinham tido um número menor de parceiros sexuais e e) A prática religiosa se mostrou como fator de proteção para os comportamentos de risco para ambos os sexos, no entanto, esse fator de proteção foi maior para as moças do que para os rapazes.

Referente ao perfil religioso observou-se que a maioria seguia afiliação religiosa e praticava sua religião. Estes resultados são similares aos observados em outros estudos brasileiros ${ }^{3,13}$ que envolveram adolescentes escolares. Nesta investigação, identificou-se também prevalências elevadas para a maioria dos comportamentos de risco à saúde, principalmente quando relacionados aos níveis insuficientes de atividade física $(64,9 \%)$ e consumo de bebidas alcoólicas $(28,6 \%)$. Estas condutas negativas têm sido observadas em estudos com adolescentes brasileiros ${ }^{14,15}$. Isso reforça a preocupação das agências de saúde com a exposição dos jovens a estes comportamentos, pois se trata de importante problema de saúde pública. Investigações ${ }^{13,16}$ indicaram que a preguiça, condições climáticas, falta de companhia, tempo e local adequado para a prática representam barreiras para a prática de atividades físicas nesta fase. Estudos ${ }^{17,18}$ revelaram que baixo custo, curiosidade, apelo social e midiático facilitam o consumo de bebidas alcoólicas.

Os dados revelaram também que adolescentes, de ambos os sexos, expostos a mais comportamentos de risco de forma simultânea, não tinham ou não praticavam uma religião. $\mathrm{Loch}^{4}$, em estudo com escolares do Ensino Médio em Florianópolis, identificou uma maior exposição aos fatores de risco à saúde entre aqueles adolescentes menos religiosos quando comparados com os mais religiosos. Deste modo, a religiosidade pode funcionar como mecanismo de resiliência, no qual o adolescente busca melhoria dos pensamentos e ações desenvolvendo uma vida saudável. As instituições religiosas conseguem proporcionar uma rede de significado, pertencimento e apoio social necessário para que o indivíduo possa incorporar ensinamentos de caráter moral capazes de dar sentido à vida e assim, facilitar as escolhas de comportamentos salutares, principalmente nesta fase da vida, como revelado no estudo de Santos et al. ${ }^{19}$.

Neste estudo foi observado que não adotar uma religião ou ter e não seguir os hábitos fomentados por ela aumenta as chances de exposição a comportamentos de risco tais como consumo de bebidas alcoólicas, drogas ilícitas, tabaco e envolvimento com dois ou mais parceiros sexuais, nos adolescentes de ambos os sexos. Resultados similares foram observados em estudos nacionais $^{3,20} \mathrm{e}$ internacionais ${ }^{21,22}$. Após observar a importância de seguir e respeitar as normas, valores e crenças pregadas por uma religião para afastar os jovens dos distintos comportamentos de risco, foi analisado se a escolha de uma determinada religião poderia estar associada a tal resultado. Neste sentido foi 
encontrado que seguir e respeitar as normas morais e os dogmas fomentados por uma religião, independentemente de qual seja essa religião, afasta os jovens de hábitos que possam denegrir sua saúde como o consumo de álcool, cigarros, drogas e um número elevado de parceiros sexuais.

Interessante notar que, as moças que praticavam uma religião tinham menos chances de exposição aos comportamentos de risco do que os rapazes que praticavam suas religiões. Resultados semelhantes foram encontrados nas investigações de $\mathrm{Caputo}^{23}$ e Pitel et al. ${ }^{24} \mathrm{~A}$ este respeito, a relação de gênero com a religião pode ser explicada por Bourdieu ${ }^{25}$, quando sugere que o babitus primário e secundário das mulheres reforça elementos subjetivos de sensibilidade e espiritualidade. Elementos estes que estão sempre em sintonia com a vivência religiosa, os quais são influenciados pelas famílias e pela Igreja desde a infância.

No presente estudo não foi observada uma associação significativa entre religiosidade e nível de atividade física. Este resultado diverge de outros estudos com adolescentes ${ }^{4,26}$ que verificaram que a religiosidade estava associada a um estilo de vida fisicamente ativo. Entretanto, estas investigações retratam uma realidade que está inserida no contexto internacional, no qual há normas e padrões religiosos distintos do cenário brasileiro.

As análises da religiosidade possibilitaram tratar o fenômeno como próprio da experiência humana e tem sido considerado um componente essencial da saúde e bem-estar ${ }^{27}$. Contudo, apenas a menção de seguir uma afiliação religiosa, não significa incorporar valores e dogmas aconselhados pela doutrina. De acordo com Marsiglia e colaboradores ${ }^{22}$, uma pessoa apenas pode ser considerada religiosa quando está envolvida em atividades relacionadas à religião como orações, adoração e participação em grupos religiosos. Neste caso, a religiosidade pode exercer certo controle sobre as atitudes e adoção de comportamentos dos indivíduos, inclusive entre os adolescentes. Assim, a religiosidade pode ser um fator que influencia a conduta dos adolescentes auxiliando na formação a percepção dos comportamentos de riscos à saúde.

Apesar de este estudo evidenciar a influência da religiosidade para a adoção de comportamentos positivos à saúde do adolescente, não podemos deixar de mencionar também o papel potencialmente negativo da religião, uma vez que determinadas doutrinas religiosas estimulam condutas contrárias à saúde do indivíduo, mas que são julgadas como positivas para quem as seguem. Seguindo esta linha de raciocínio, as pesquisas de Araújo e Vieiralves-Castro ${ }^{28}$ e Grois$\operatorname{man}^{29}$ mostraram que alguns grupos étnicos como o Santo Daime e indígenas, por exemplo, fazem uso de bebidas, plantas e outras substâncias consideradas psicoativas em suas práticas ritualísticas, com a crença de que diversos males físicos, mentais e espirituais podem ser curados. Outro exemplo está relacionado à sexualidade, em que algumas congregações religiosas se mostram contra ao controle da natalidade e de uso de métodos anticoncepcionais por estar associado à devassidão sexual, como apresentado no estudo de Dantas ${ }^{30}$. Estes exemplos, entre outros, revelam o quanto é complexo discutir esta temática que, por vezes, é apresentado apenas o lado positivo da religião, perdendo de vista o seu papel negativo no comportamento relacionado à saúde do indivíduo.

Algumas limitações devem ser consideradas no presente estudo. Primeiro, não foram considerados aqueles que estudavam na rede privada. Segundo, este estudo considerou apenas o número de parceiros sexuais para determinar a adoção de comportamento de risco à saúde. Entretanto, seria interessante ava- 
liar também a não utilização de preservativos em todas as relações sexuais anteriores, mas tal informação não foi coletada. Vale salientar que tanto a relação sexual desprotegida como a iniciação sexual precoce podem ser considerados como comportamentos de risco. Outra limitação foi ter utilizado instrumento fechado para investigar o aspecto da religiosidade. Tratando-se de uma temática complexa, seria necessária uma maior exploração da subjetividade dos adolescentes, por meio de roteiros abertos de entrevistas, com o intuito de investigar a importância da religiosidade em suas vidas e como a religião os protege dos comportamentos de risco à saúde. Outro ponto foi a medida da atividade física que foi auto-referida Há também a possibilidade de causalidade reversa como uma característica inerente de estudos transversais. A fim de atenuar essas limitações, a coleta de dados foi realizada por uma equipe previamente treinada. Mesmo ciente das limitações inerentes ao uso do questionário, os indicadores de reprodutibilidade apresentaram coeficiente de correlação intraclasse de moderados a altos na maioria dos itens do questionário.

Entre os pontos fortes da presente pesquisa, pode-se destacar não só a representatividade da população de estudantes das escolas da rede pública do ensino médio do Estado de Pernambuco, mas também o resultado encontrado da associação entre a prática religiosa e os distintos comportamentos de risco, após o ajustamento pelas diferentes afiliações religiosas, respeitando as diferenças entre os sexos e controlando as possíveis variáveis de confusão.

A partir deste estudo, foi possível concluir que a religiosidade, independentemente da religião seguida, mostrou-se associada à proteção aos comportamentos de risco à saúde neste grupo de adolescentes. Futuros estudos precisam ser conduzidos na tentativa de compreender com profundidade o papel da afiliação e prática religiosa como aspectos moduladores na vida dos adolescentes, como por exemplo, aspectos particulares entre as religiões e os tipos de práticas religiosas.

\section{Financiamento}

Conselho Nacional de Desenvolvimento Científico e Tecnológico (financiamento da pesquisa)

\section{Agradecimento}

Coordenação de Aperfeiçoamento de Pessoal de Nível Superior - CAPES (Bolsa de mestrado)

\section{Contribuições individuais de cada autor}

Ana Raquel Mendes dos Santos responsabilizou-se pela concepção, redação e revisão crítica do artigo; Luciano Machado Ferreira T. Oliveira responsabilizouse pela análise e interpretação dos dados, redação, revisão crítica do artigo e aprovação da versão a ser publicada; Fosé Cazuza de Farias fúnior responsabilizou-se pela interpretação dos dados, revisão crítica do artigo e aprovação da versão a ser publicada; Priscilla Pinto Costa da Silva responsabilizou-se pela redação e revisão crítica do artigo e aprovação da versão a ser publicada; Emília Pinto Costa da Silva responsabilizou-se pela redação e revisão crítica do artigo e aprovação da versão a ser publicada; e Clara Maria Silvestre Monteiro de Freitas revisão crítica do artigo e aprovação da versão a ser publicada. 


\section{REFERÊNCIAS}

1. Teófilo DDN, Junqueira SRA. Religiosidade na adolescência: a necessidade de uma construção consciente e social. Rev Pistis Prax Teol Pastor. 2011;3(2):635-52.

2. Pinto EB. Espiritualidade e Religiosidade: articulações. REVER 2009 dez:68-83.

3. Bezerra J, Barros MVG, Tenório MCM, Tassitano RM, Barros SSH, Hallal PC. Religiosidade, consumo de bebidas alcoólicas e tabagismo em adolescentes. Rev Panam Salud Publica. 2009;26(5):440-6.

4. Loch MR. Comportamentos relacionados à saúde e indicadores de religiosidade em adolescentes escolares [dissertação de mestrado]. Santa Catarina: Universidade Federal de Santa Catarina; 2006.

5. Araújo AC, Lunardi VL, Silveira RS, Thofehrn MB, Porto AR. Relacionamentos e interações no adolescer saudável. Rev Gaúcha Enferm. 2010;31(1):136-42.

6. Ministério da Saúde (Brasil), Secretaria de Atenção à Saúde, Departamento de Ações Programáticas Estratégicas. Diretrizes nacionais para a atenção integral à saúde de adolescentes e jovens na promoção, proteção e recuperação da saúde: Ministério da Saúde; 2010.

7. Andrade SSCA, Yokota RTC, Sá NNB, Silva MMA, Araújo WN, Mascarenhas MDM, et al. Relação entre violência física, consumo de álcool e outras drogas e bullying entre adolescentes escolares brasileiros. Cad Saúde Pública. 2012;28(9):1725-36.

8. Tucker JS, Ryan GW, Golinelli D, Ewing B, Wenzel SL, Kennedy DP, et al. Substance Use and Other Risk Factors for Unprotected Sex: Results From An Event-Based Study of Homeless Youth. AIDS Behav. 2012;16(6):1699-707.

9. Mélo EM, Meneses AS, Silva Júnior AGS, Wanderley Júnior RS, Barros MVG. Associação entre religiosidade, atividade física e comportamento sedentário em adolescentes. Rev Bras Ativ Fis e Saúde. 2012;17(5):359-69.

10. Prochaska JJ, Sallis JF, Long B. A physical activity screening measure for use with adolescents in primary care. Arch Pediatr Adolesc Med. 2001;155(5):554-9.

11. World Health Organization (WHO). Global Recommendations on Physical Activity for Health; 2010 [Acesso em 2013 jan 15]. Disponível em: http://www.who.int/ dietphysicalactivity/factsheet_recommendations/en/.

12. Cruzeiro ALS, Souza LDM, Silva RA, Pinheiro RT, Rocha CLA, Horta BL. Comportamento sexual de risco: fatores associados ao número de parceiros sexuais e ao uso de preservativo em adolescentes. Ciênc saúde coletiva. 2010;15(1):1149-58.

13. Santos MS, Fermino RC, Reis RS, Cassou AC, Añez CRR. Barreiras para a prática de atividade física em adolescentes. Um estudo por grupos focais. Rev Bras Cineantropom Desempenho Hum. 2010;12(3):137-43.

14. Campos JADB, Almeida JC, Garcia PPNS, Faria JB. Consumo de álcool entre estudantes do ensino médio do município de Passos - MG. Ciênc saúde coletiva. 2011;16(12):4745-54.

15. Farias Júnior JC, Nahas MV, Barros MVG, Loch MR, Oliveira ESA, De Bem MFL, et al. Comportamentos de risco à saúde em adolescentes no Sul do Brasil: prevalência e fatores associados. Rev Panam Salud Publica. 2009;25(4):344-52.

16. Copetti J, Neutzling MB, Silva MC. Barreiras à prática de atividades físicas em adolescentes de uma cidade do sul do Brasil. Rev Bras Ativ Fis e Saúde. 2010;15(2):88-94.

17. Mendes LR, Teixeira MLO, Ferreira MA. Bebida alcohólica en la adolescencia: el cuidado-educación como estrategia de acción de la Enfermería. Esc. Anna Nery Rev Enferm. 2010;14(1):158-64.

18. Silva SED, Padilha MI. Atitudes e comportamentos de adolescentes em relação à ingestão de bebidas alcoólicas. Rev Esc Enferm USP. 2011;45(5):1063-9.

19. Santos ARM, Silva EAPC, Silva PPC, Cartaxo HGO, Freitas CMSM. Estilo de vida na adolescência: o envolvimento religioso atuando nos comportamentos de risco à saúde. Pensar prát (Impr). 2014;17(1):14-25.

20. Dalo L, Martins RA. Uso de álcool entre adolescentes escolares: um estudo piloto. Paidéia. 2011;21(50):329-34.

21. Edwards LM, Haglund K, Fehring RJ, Pruszynski J. Religiosity and sexual risk behaviors among latina adolescents: trends from 1995 to 2008. J Womens Health (Larchmt). 2011;20(6):871-77. 
22. Marsiglia FF, Kulis S, Nieri T, Parsai M. God Forbid! Substance Use Among Religious and Nonreligious Youth Am J Orthopsychiatry. 2005;75(4):585-98.

23. Caputo, RK. Religiousness and adolescent behaviors: a comparison of boys and girls. J Relig Spiritual Soc Work. 2005;24(3):39-67.

24. Pitel, L., Geckova AM, Kolarcik P, Halama P, Reijneveld SA, Dijk JPV. Gender differences in the relationship between religiosity and health-related behaviour among adolescents. J Epidemiol Commun H. 2012;66:1122-8.

25. Bourdieu P. O Poder Simbólico. Rio de Janeiro: Bertrand Brasil, 2000.

26. Leopold D, Juniu S. Incorporating a spiritual component into the health education aspects of a physical (activity) education program. Physical Educator. 2008;65(4):208-21.

27. Huang CY, Hsu MC, Chen TJ. An exploratory study of religious involvement as a moderator between anxiety, depressive symptoms and quality of life outcomes of older adults. J Clin Nurs. 2011;21(5-6):609-19.

28. Araújo MCR, Vieiralves-Castro R. O Uso Ritual das Plantas de Poder. Horiz Antropol (Online). 2007;13(27):358-62.

29. Groisman A. Missão e Projeto: motivos e contingências nas trajetórias dos agrupamentos do Santo Daime na Holanda. Plura. 2004;4(1):1-18.

30. Dantas BSA. Sexualidade, cristianismo e poder. Estud pesqui psicol. 2010;10(3):700-28.

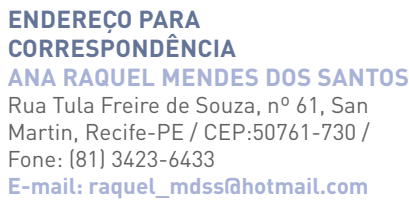

